\section{MEZCLA SOCIAL E INTEGRACIÓN URBANA: APROXIMACIONES TEÓRICAS Y DISCUSIÓN DEL CASO CHILENO ${ }^{1}$}

Javier Ruiz-Tagle y Scarlet Romano $^{3}$

\section{Resumen}

La mezcla social, en términos generales, denota la diversidad social de un área geográfica determinada, sea esta económica, racial, étnica, cultural, etc. La mezcla social es un viejo y persistente ideal de planificación (marcada por pretensiones normativas), ha sido lograda a través de diferentes medios, ha sido propuesta para lograr una amplia variedad de objetivos, y ha sido usada intercambiablemente para referirse a conceptos como 'integración', 'comunidades de ingresos diversos', 'desconcentración de la pobreza', 'comunidades balanceadas', etc. Ha habido abundante literatura desde los años 90, a tal punto que es uno de las materias más estudiadas en los estudios urbanos.

\section{SOCIAL MIX AND URBAN INTEGRATION: THEORETICAL APPROACHES AND DISCUSSION OF THE CHILEAN CASE'}

Javier Ruiz-Tagle ${ }^{2}$ y Scarlet Romano ${ }^{3}$

\section{Abstract}

Social mix, in general terms, denotes the social diversity of a certain geographic area, being this economic, racial, ethnic, cultural, etc. Social mix is an old and persistent ideal of planning (marked by normative pretensions), has been achieved through different means, has been proposed to reach a wide variety of goals, and has been used interchangeably to refer to concepts like 'integration', 'mixed-income communities', 'poverty deconcentration', 'balanced communities', etc. There has been abundant literature from the 1990s, to the extent that it is one of the most researched topics in urban studies. At present, the discussion on social mix revolves around five key concepts: contact hypothesis, 
En la actualidad, la discusión sobre mezcla social gira alrededor de cinco conceptos claves: hipótesis del contacto, redes sociales, control social, modelos de rol y geografías de la oportunidad. En Chile, desde el año 2006 ha habido políticas de mezcla social, con la expectativa de que la proximidad física entre diferentes grupos sociales podría facilitar la integración. En este artículo revisamos el concepto de mezcla social desde una perspectiva histórica y comparativa, y luego estudiamos las políticas chilenas bajo los cinco conceptos mencionados, mostrando la poca eficacia que han tenido los proyectos de integración en cumplir los objetivos propuestos.

\section{PALABRAS CLAVE: MEZCLA SOCIAL; INTEGRACIÓN SOCIAL; PLANIFICACIÓN URBANA; POLÍTICAS PÚBLICAS}

\author{
Recibido: 15-08-2018
}

Aceptado: 21-03-2019

1 Fondecyt de Iniciación 11150426; Fondo de Inserción de Capital Humano Avanzado en la Academia (Conicyt Folio 79150032); Centro de Desarrollo Urbano Sustentable, CEDEUS (Proyecto Fondap 15110020).

2 Chile. Instituto de Estudios Urbanos y Territoriales, Facultad de Arquitectura, Diseño y Estudios Urbanos, Pontificia Universidad Católica de Chile, http://orcid.org/0000-0002-9803-9215. Correo electrónico: jaruiz-tagle@uc.cl

3 Alemania. Hochschule für Technik und Wirtschaft Berlin - HTW y Metropolia University of Applied Sciences, Finland, http://orcid.org/0000-0002-0279-5498. Correo electrónico: scarlet.arq@ gmail.com social networks, social control, role models, and geographies of opportunity. In Chile, from 2006 there have been social mix policies, with the expectation that physical proximity between different social groups could facilitate integration. In this article we review the concept of social mix from a historical and comparative perspective, and then we study Chilean policies under the five mentioned concepts, showing the low efficacy that these projects have had in meeting the proposed goals.

\section{KEYWORDS: SOCIAL MIX, SOCIAL INTEGRATION, URBAN PLANNING, PUBLIC POLICIES}

\author{
Received: 15-08-2018
}

Accepted: 21-03-2019

1 Fondecyt Iniciation project No 11150426; Insertion of Advanced Human Capital Fund (Conicyt No 79150032); Center for Sustainable Urban Development, CEDEUS (Fondap project No 15110020.)

2 Chile. Institute of Urban and Territorial Studies, Faculty of Architecture, Design and Urban Studies, Pontifical Catholic University of Chile, http://orcid.org/0000-0002-9803-9215. Email: jaruiztagle@uc.cl.

3 Germany. Hochschule für Technik und Wirtschaft Berlin - HTW y Metropolia University of Applied Sciences Finland, http://orcid. org/0000-0002-0279-5498. Email: scarlet.arq@gmail.com 


\section{Introducción}

La mezcla social ha sido largamente discutida e implementada en la planificación urbana, lo que no quita que sea un concepto altamente confuso (Galster, 2013). En términos generales, el término denota la diversidad social de un área geográfica determinada, la que puede ser económica, racial, étnica, cultural, entre otras. El debate sobre este concepto ha estado presente en la academia y en el desarrollo de políticas públicas abordando no solo su definición teórica, sino también preguntas tales como su finalidad, los instrumentos para su implementación y su efectividad. En la actualidad, la discusión sobre mezcla social gira alrededor de cinco conceptos claves: hipótesis del contacto, redes sociales, control social, modelos de rol y geografías de la oportunidad.

En Chile, desde el año 2006 la Política UrbanoHabitacional de Integración Social ha impulsado el desarrollo de proyectos de vivienda con mezcla social, tales como los Proyectos de Integración Social (PIS), los Proyectos de Reactivación Económica con Integración (PREIS) y los Proyectos de Integración Social y Territorial (PIST). Estos han puesto en práctica la premisa existente a nivel internacional sobre la mezcla social, que postula que la proximidad física entre diferentes grupos sociales podría facilitar la integración social (Ruiz-Tagle, 2013). Sin embargo, actualmente existen numerosos estudios sobre estos proyectos que ponen en duda su eficacia frente a esta premisa.

Este artículo tiene un objetivo doble: primero, discutir los orígenes y la concepción actual sobre la mezcla social, profundizando en sus objetivos e instrumentos de implementación. Segundo, evaluar los resultados de ocho estudios existentes realizados por entidades académicas (seis documentos, entre artículos y conferencias) y gubernamentales (una cuenta pública y un informe de evaluación realizado para la Dirección de Presupuesto) de los PIS y los PREIS en Chile 4 . Para responder a estos objetivos, primero se revisa el concepto de mezcla social en los estudios urbanos desde una perspectiva histórica y comparativa. Y segundo, se estudian las políticas aplicadas en Chile, discutiendo los resultados de todas las evaluaciones parciales que hasta ahora se han desarrollado de los PIS y PREIS, bajo los cinco conceptos claves antes mencionados.

4 Los PIST no se consideran en este artículo debido a que no existen estudios que analicen su funcionamiento. 


\section{El concepto de mezcla social en los estudios urbanos}

La mezcla social es un viejo y persistente ideal de planificación (marcada por pretensiones normativas), que ha sido lograda a través de diferentes medios, ha sido propuesta para lograr una amplia variedad de objetivos, y ha sido usada intercambiablemente para referirse a conceptos como 'integración', 'comunidades de ingresos diversos', 'desconcentración de la pobreza', 'comunidades balanceadas', etc. Se ha producido abundante literatura desde los años 90 al respecto, a tal punto que la mezcla social es uno de las materias más estudiadas en los estudios urbanos, con al menos cinco números especiales en destacadas revistas académicas dedicadas al tema: Urban Studies (2008, 45:7), Housing Studies (2010, 25:2), Cities (2013, 35), Cityscape (2013, 15:2), e International Journal of Urban and Regional Research (2014, 38:4). Aunque la evidencia sobre los efectos de la mezcla social es diversa, la cantidad y variedad de problemas creados han llevado a que la gran mayoría de la academia se oponga a esta idea (Bolt y van Kempen, 2013).

Hemos observado en la literatura internacional al menos siete medios a través de los cuales se ha logrado establecer la mezcla social en el espacio urbano. Los primeros cuatro corresponden a políticas públicas de diferente naturaleza y los últimos tres son solo procesos urbanos emergentes. Primero, y la más destacada, hay políticas públicas destinadas a re-desarrollar conjuntos de vivienda social existentes y deteriorados. Este proceso usualmente involucra la demolición de algunas (o todas las) unidades de vivienda social, el desplazamiento de residentes pobres, la venta de unidades a residentes nuevos de mayor estatus, y la relocalización de solo algunos de los residentes antiguos después de un proceso de filtro. Hasta ahora, estas políticas han sido aplicadas en países europeos (Atkinson y Kintrea, 2000; Kleinhans, 2004; Musterd y Andersson, 2005), en Estados Unidos (Goetz, 2013), en Canadá (August, 2008) y en Australia (Arthurson, 2002). Como puede adivinarse, este ha sido el tipo más controversial y criticado de mezcla social.

Segundo, hay políticas de desegregación que han insertado hogares de bajo estatus en barrios de alto estatus, anteriormente segregados. Esto ha sido aplicado a través de políticas enfocadas en los hogares, en donde se les dan vouchers y asistencia a las familias para que se cambien de vivienda, con ejemplos en algunas ciudades de Estados Unidos (Deluca y Rosenbaum, 2010). Y también ha sido aplicado a través de incentivos de regulación urbana que permiten mayor densidad a nuevos desarrollos que contengan un porcentaje de vivienda social, lo que ha sido implementado en Estados Unidos (Hananel, 2014), en Brasil (Budds y Teixeira, 2005) y en Colombia (Lobo, 2015). 
Tercero, hay regulaciones de planificación del uso de suelo que obligan la construcción de proyectos de vivienda social en nuevos terrenos, en donde los diferentes grupos sociales son todos nuevos residentes de un área no desarrollada. Hay ejemplos de esto en Australia (Ruming, Mee, y McGuirk, 2004), en algunos estados de Estados Unidos (Calavita y Grimes, 1998), y en Chile (Siclari, 2009), a través de proyectos de vivienda con mezcla social, que abordaremos más adelante. Dentro de este tipo, hay otras herramientas de planificación que mandatan cesiones de terreno para construir viviendas sociales dentro del desarrollo condicionado de grandes paños de terreno, en España (Muñoz y Korthals, 2007) y en Chile (Borsdorf e Hidalgo, 2008).

Cuarto, hay prohibiciones para mudarse hacia un área específica, o cuotas de grupos de bajo estatus, en ciertos barrios: ya sea para prevenir concentraciones étnicas o de pobreza, o para promover la inclusión de estos grupos en áreas segregadas de mayor estatus. Esto ha sido aplicado en Alemania (Bolt, 2009) y en Francia (Bacqué, Fijalkow, Launay y Vermeersch, 2011).

Más allá de las políticas públicas, la mezcla social también ha sido lograda por otros procesos. Quinto, áreas de mezcla social han sido creadas como resultado de la colonización de barrios cerrados de alto estatus en zonas pobres, a partir de sofisticadas estrategias desde los desarrolladores inmobiliarios para capturar altas rentas. Esto ha ocurrido en Chile (Salcedo y Torres, 2004), Argentina (Svampa, 2001) y Brasil (Caldeira, 2000). Sexto, algunos patrones de diversidad estable han aparecido como resultado de cambios socio-demográficos y/o de mercado, a menudo durante procesos de gentrificación o de escape de blancos de clase media ('white flight'). Esto ha sido observado en algunas ciudades de Estados Unidos (Ellen, 2000; R. Smith, 1998) y en Sudáfrica (Lemanski, 2006). Finalmente, la mezcla social se ha construido por la influencia activa de organizaciones de base que trabajan para generar una coexistencia deseada. Como proceso para lograr diversidad social, este es uno de los más positivos, pero también es uno de los menos estudiados, con tan solo unos pocos casos documentados en Estados Unidos (Cashin, 2004; Nyden, Maly y Lukehart, 1997).

A pesar de la creciente cantidad de literatura en décadas recientes, la mezcla social ha sido un ideal de la planificación urbana por casi doscientos años. Los orígenes de la idea se remontan a la era victoriana en Inglaterra, cuando la mezcla social era respaldada para diferentes propósitos, desde las nostalgias románticas de los pueblos pequeños, a los remedios utilitarios para el hacinamiento industrial (Arthurson, 2012a; Sarkissian, 1976; Sarkissian, Forsyth, y Heine, 1990). Otros partidarios incluían Ebenezer Howard, Octavia Hill y Lewis Mumford. Howard estaba a favor de las 
micro-segregaciones dentro de las más heterogéneas Ciudades Jardín. Hill propuso la idea de un 'espíritu de emulación' (similar a los 'modelos de rol' que propone la literatura estadounidense desde los 90), que permitiría a los pobres aprender de sus vecinos de mayor estatus. Y Mumford defendía la mezcla social como una función esencial de la ciudad, lo que permitiría una fertilización entre culturas (Sarkissian, 1976). Después de la Segunda Guerra Mundial, la idea de la mezcla social revivió. Las ideologías de un mundo sin clase llegaron a la planificación urbana, con un foco en la regeneración de la vida comunal y en la remoción de barreras bajo las nuevas economías de bienestar (Sarkissian, 1976). En Estados Unidos, Jane Jacobs celebró la vitalidad de los barrios heterogéneos, cercana a la idea de las antiguas aldeas. Luego, dos hitos marcaron los 90 en Estados Unidos: la reentrada del diseño urbano y el renacimiento de la mezcla social. El Nuevo Urbanismo fue un movimiento que quiso participar en el desarrollo de las comunidades de ingresos diversos del programa HOPE VI, contribuyendo con sus prescripciones normativas, y re-introduciendo el diseño urbano en la discusión de la planificación (J. Smith, 2006). Al mismo tiempo, después de varios años de silencio provocado por las críticas de integración forzada desde la izquierda afroamericana, la mezcla social reapareció como una nueva sabiduría consensual de los debates de planificación urbana, con una retórica política que aun apunta a los ideales progresistas históricos de igualdad de oportunidades, pero con una práctica que ha estado más en línea con la gobernanza neoliberal (August, 2008).

El concepto de mezcla social es altamente confuso (Galster, 2013), debido a que está fundamentalmente basado en pretensiones normativas, más allá de certezas empíricas significativas. En efecto, las preguntas más frecuentes respecto de la mezcla social son acerca de su efectividad, pero la variedad de objetivos para los cuales esta política ha sido implementada impiden una respuesta definitiva. Sarkissian (1976) observó nueve diferentes -y algunos incoherentes- objetivos para apoyar la mezcla social: espíritu de emulación, mejor diversidad y estándares estéticos, fertilización entre culturas, igualdad de oportunidades, armonía social a través de la reducción de tensiones sociales y raciales, conflicto social para incentivar la madurez individual y social, mejor funcionalidad física de la ciudad, estabilidad de áreas residenciales, y reflejo de la diversidad del mundo moderno. En el presente, el debate sobre la mezcla social gira alrededor de cinco conceptos claves, los que a su vez representan los principales fundamentos de las políticas públicas.

Primero, la llamada hipótesis del contacto asume que el contacto interpersonal es un mecanismo efectivo para reducir el prejuicio entre distintos grupos sociales (Allport, 1954; Dixon, 2001). Dado que el prejuicio se basa en la ignorancia sobre 'el otro', el 
contacto podría permitir algún grado de conocimiento, y como resultado, mejores prospectos de entendimiento y aceptación. La crítica a esto incluye la posibilidad del 'contacto negativo' ('deterioro ambiental' o 'hipótesis del conflicto'), que implica el desapego de dinámicas entre grupo y procesos persistentes de construcción de límites.

Segundo, la idea de redes sociales como capital social, enfatiza la importancia del retorno de las clases medias a los espacios centrales de la ciudad. Al ocurrir esto, la mezcla social facilitaría el desarrollo de 'lazos débiles' a través de los cuales los habitantes de bajos ingresos podrían acceder a redes de empleo y otros recursos (Joseph, 2006). La crítica más obvia a este supuesto es que los individuos de alto estatus no estarían dispuestos a compartir sus recursos y sus redes con los pobres tan fácilmente.

Tercero, la idea de control social indica que la presencia de habitantes de altos ingresos llevaría a mejores comportamientos respecto de las normas, y a un mayor orden y seguridad (Joseph, 2006). La crítica apunta aquí a la idea unilateral de que los pobres estarían mejor controlados por individuos más acomodados en su propio barrio, y no por ellos mismos (Tach, 2009).

Cuarto, la idea de los modelos de rol implica que la presencia de vecinos de alto estatus llevaría a la adopción de comportamientos socialmente aceptables y constructivos, es decir, la asimilación de una cultura de clase media (Joseph, 2006). Similar a lo anterior, esta idea es criticada por asumir que los pobres estarían mejor orientados en su comportamiento y objetivos por residentes de mayor estatus, y que sus valores y costumbres son intrínsecamente negativos (Smith, 2006, 2010).

Finalmente, la geografía de la oportunidad apunta a la generación de una nueva demanda de mercado y mayor presión política, atrayendo bienes y servicios de mayor calidad (Howell-Moroney, 2005; Joseph, 2006), lo que a su vez influencia las perspectivas de vida de los residentes locales (Galster y Killen, 1995). Esta idea tiene un supuesto implícito orientado al mercado, el cual sugiere que la localización de las oportunidades sigue a los grupos más poderosos, sin tomar en cuenta la economía política de las instituciones en la redistribución de recursos, dejando así a la mezcla social como la 'única' alternativa para mejorar las perspectivas de vida de los pobres.

Como consecuencia de la amplia variedad de objetivos planteados para la mezcla social se han desarrollado muchos estudios que se enfocan en distintas dimensiones y problemas de este. En términos físicos, hay varias investigaciones sobre el diseño físico para la mezcla social (Greenbie, 1976; Talen, 2008; Vischer, 1986a, 1986b), y sobre la percepción de los impactos físicos de la transformación de viviendas sociales en comunidades de ingresos diversos (Joseph y Chaskin, 2010). En términos funcionales, hay estudios que se enfocan 
en las redes sociales de los grupos de bajo estatus (Atkinson y Kintrea, 2000), en los beneficios generales de la mezcla social para los pobres (Fraser y Kick, 2007), en los efectos de la mezcla respecto del estatus socioeconómico, los ingresos y el empleo (Galster, Andersson, y Musterd, 2010; Galster, Andersson, Musterd, y Kauppinen, 2008; Sarkissian et al., 1990; Van Ham y Manley, 2010), en los efectos en los valores de las propiedades y la calidad de las escuelas (Sarkissian et al., 1990), y en la atracción de equipamientos para grupos sociales específicos (Atkinson y Kintrea, 2000). En términos relacionales, hay estudios que se enfocan en la inclusión y cohesión general entre los diferentes grupos sociales (Arthurson, 2002; Atkinson y Kintrea, 2000; Chaskin y Joseph, 2010; Joseph, 2008; Sarkissian et al., 1990), en las interacciones sociales, la organización social y el control social (Joseph y Chaskin, 2010; Kleit, 2005; Rosenbaum, Stroh, y Flynn, 1998; Tach, 2009), en la participación y deliberación de los diferentes grupos en juego (Chaskin y Joseph, 2010; Chaskin, Khare, y Joseph, 2012), y en las percepciones sobre seguridad (Rosenbaum et al., 1998). En términos simbólicos, hay estudios sobre las imágenes, reputación y estigmatización de las áreas socialmente mixtas (Arthurson, 2010, 2012b, 2013; August, 2014). Y, finalmente, hay varios trabajos que estudian la idea general de la mezcla social y los esfuerzos de planificación urbana alrededor de ella, con foco en las consecuencias, potencialidades, beneficios para los actores, grado de satisfacción, la estabilidad futura, etc. (Arthurson, 2002; Fraser y Kick, 2007; Joseph, 2008; Rosenbaum et al., 1998; Sarkissian et al., 1990).

Algunos autores dicen que la evidencia sobre el tema es diversa. Pero dado que esta es una política basada en las creencias (Cheshire, 2007), hay una clara división entre aquellos que la apoyan (funcionarios públicos y centros de estudios) y aquellos que se oponen (la academia) (Bolt y van Kempen, 2013). En Estados Unidos, think tanks liberales, como el Brookings Institution y el Urban Institute, han apoyado de manera general los programas de mezcla social como el HOPE VI, adhiriendo y contribuyendo a un consenso general sobre la dispersión de la pobreza y la mezcla social (Arthurson, 2012a; Imbroscio, 2008). Hipótesis tempranas sobre este programa esperaban efectos sobre el acceso al empleo, la auto-suficiencia y la calidad de vida entre los pobres (Fraser, Oakley, y Levy, 2013). Cisneros (2009) sostiene que la mezcla social es beneficiosa para las oportunidades laborales, para proveer vivienda adecuada para los pobres y para las economías metropolitanas. En una revisión extensa, Galster (2013) afirma que la idea de la mezcla social puede justificarse en dos objetivos: para la equidad, de modo de mejorar el bienestar de los pobres, evitando su concentración y promoviendo la diversidad; y para la eficiencia, de modo de mejorar el bienestar de la sociedad en 
su conjunto, pero solo con bajos porcentajes de población pobre en las áreas de mezcla.

La crítica sin embargo, en cantidad de argumentos, evidencia y publicaciones, es bastante contundente. En términos socio-culturales, las políticas de mezcla social han sido criticadas por forzar la asimilación, quiebre de lazos, atomización, pérdida de capital social, alienación, exclusión de los 'peores' residentes, y adopción de reglas y comportamientos unilaterales entre los grupos de bajo estatus (Bolt y van Kempen, 2013; Bolt, Özüekren, y Phillips, 2010; Greenbaum, 2008; Smeets y Salman, 2008). Además, los críticos enfatizan que los esperados procesos de modelamiento de roles desde los residentes de alto estatus, han terminado en prácticas de 'otredad', estigmatización y opresión (Ruming et al., 2004). Más aún, se señala que los barrios socialmente mixtos no son mejores que otros barrios, debido a que no hay efecto en la cohesión social, hay una baja interacción entre grupos y un bajo apego al lugar, y ofrecen muy pocos beneficios para reducir problemas (Bolt y van Kempen, 2013).

En términos político-económicos, si bien hay acuerdo en que la mezcla social provee un mejoramiento físico significativo respecto de las condiciones previas, los críticos recalcan que la pobreza y las relaciones sociales permanecen intactas (Silver, 2013). De hecho, muchos dicen que la mezcla social no se dirige al problema más importante (la pobreza en sí misma y no su concentración física), argumentando que esta solo trata un síntoma, y que ha sido ineficaz en contrarrestar la auto-segregación (Bolt, Phillips, y Van Kempen, 2010; Bolt y van Kempen, 2013; Cheshire, 2007).

Del mismo modo, cuando la mezcla social ha sido lograda a través de demoliciones, ha habido reducciones en el parque de viviendas y en el sector de viviendas sociales en general, aumentos en los costos de vida, y desplazamiento de residentes pobres y minorías raciales para abrir espacio a residentes de alto estatus (Bolt et al., 2010; Bolt y van Kempen, 2013; Goetz, 2013; Smeets y Salman, 2008). La crítica más radical concibe la mezcla social como una estrategia de reinversión de capital a través de la regulación, marginalización y el desplazamiento de los pobres (Fraser et al., 2013). En otras palabras, la mezcla social sería un proceso de re-desplazamiento de clase, a través del cual se extrae renta y se controla socialmente a los pobres (Bridge, Butler, y Le Galès, 2014; Uitermark, 2014). En un nivel macro-social, finalmente, si bien la mezcla social acarrea un simbolismo poderoso de desegregación, hay una discrepancia considerable entre su retórica aparentemente progresista y los procesos contemporáneos de reducción del bienestar social, fragmentación social y desigualdades crecientes (Bolt et al., 2010; Ruiz-Tagle, 2016b). A pesar de todo esto, se podría esperar que esta política continúe creciendo y expandiéndose en el mundo, dada la alta popularidad que tiene entre 
funcionarios públicos, y la persuasión de su retórica en la opinión pública. Y así ha ocurrido en Chile en la actualidad.

\section{Las políticas de mezcla social en Chile}

Para describir exhaustivamente la implementación de políticas en Chile, se revisaron todas las evaluaciones desarrolladas hasta fines de 2018 en el país. El total incluyó seis documentos académicos (artículos y presentaciones en conferencias) y dos documentos gubernamentales (una cuenta pública y un informe de evaluación realizado para la Dirección de Presupuesto). Debido a que las políticas de mezcla social en Chile llevan muy poco tiempo desde su inicio, y que su implementación hasta ahora no ha sido masiva (sino más bien la excepción), tanto el número de proyectos existentes como el número de evaluaciones es bastante acotado, lo que permitió abarcar todas las evaluaciones parciales existentes que se han desarrollado de los PIS y los PREIS en Chile. Los documentos académicos analizados se enfocan en dos temáticas. Primero, se cuestiona la relevancia de las políticas habitacionales que dieron origen a los PIS y los PREIS, con evidencia internacional, y la real aplicabilidad y resultados de la lógica de mezcla social en Chile. Y segundo, se estudian los PIS y los
PREIS ya construidos y habitados, desde la perspectiva de la convivencia y de la percepción de los habitantes sobre sus conjuntos habitacionales. Los documentos gubernamentales, por su parte, están basados en el cruce de información oficial: por ejemplo, decretos, resoluciones e informes del MINVU, la Encuesta de Caracterización Socioeconómica Nacional (CASEN), estudios de la División de Política Habitacional (DPH) del MINVU, del Instituto Nacional de Estadísticas (INE), etc. El foco de estos está en el análisis del porcentaje de cumplimiento de lo establecido por los PIS y los PREIS, y no en el cuestionamiento de su objetivo o coherencia con el escenario nacional. A partir de estos dos tipos de documentos, clasificamos y discutimos los resultados observados desde la óptica de los cinco conceptos claves que actualmente están presentes en la discusión de la mezcla social en la literatura internacional: hipótesis del contacto, redes sociales, control social, modelos de rol y geografías de la oportunidad.

Hacia comienzos del año 2006, el gobierno de Michelle Bachelet se encuentra con un clima de fuerte crítica respecto de los impactos de las políticas de vivienda de los últimos 25 años. Por un lado, se reconocen los grandes logros respecto de épocas anteriores; el fin de las ocupaciones ilegales, la dotación de servicios básicos a casi toda la población, la reducción del déficit de vivienda, y la focalización hacia los más pobres (Ducci, 1997). Pero por otro lado, se revelan dos grandes problemas que se 
acumulan en este período: la mala calidad de la vivienda y los barrios construidos, y los altos niveles de segregación residencial, ambos consecuencia de una política de construcción masiva de viviendas sociales, basada en el apoyo estatal hacia el sector privado (Hidalgo, 2004; Sabatini, Cáceres, y Cerda, 2001; Sugranyes, 2005). Así, el propio Ministerio de la Vivienda y Urbanismo (MINVU) reconocía esta situación como una crisis de "buena cobertura con mala calidad".

El concepto de segregación residencial refiere a una relegación de grupos discriminados hacia áreas separadas dentro de las ciudades, ejercida en su mayoría por parte del Estado (Marcuse, 1997; Wacquant, 1997; Wirth, 1927). Si bien la segregación apunta inicialmente hacia el espacio social, como desigual distribución de grupos sociales, también puede haber una segregación del 'espacio institucional': esto es, una desigual distribución y calidad de las instituciones que atienden a la población. En contextos neoliberales como el chileno, la situación socioeconómica de los habitantes de un barrio influencia fuertemente la cantidad y calidad de recursos, oportunidades y servicios de este. Es decir, la segregación del espacio social ha sido acompañada de una segregación del 'espacio institucional'. Dicho en términos de las políticas del MINVU, el proceso de segregación residencial que resultó de la localización periférica de la vivienda social, fue aparejado de un proceso de crecientes desigualdades territoriales producidas por el sistema de impuestos territoriales, la municipalización de la salud y la educación, la re-subdivisión comunal de 1981, las políticas sociales focalizadas, etc. todos parte de las reformas neoliberales. De esta manera, se dio forma a la mala calidad generalizada de los barrios en donde se localizaron las viviendas sociales de las últimas décadas.

En este contexto, los conceptos e instrumentos vinculados a las políticas de mezcla social en Chile pueden ser organizados en dos etapas. La primera está vinculada con la Política Urbano-Habitacional de Mejoramiento de la Calidad e Integración Social de 2006, del primer gobierno de Michelle Bachelet. Esta se propuso trabajar los conceptos de integración y cohesión social, y dio origen a las primeras iniciativas: los llamados Proyectos de Integración Social (PIS). Y la segunda etapa corresponde a la Política Nacional de Desarrollo Urbano, Ciudades Sustentables y Calidad de Vida de 2014, promulgada en el primer gobierno de Sebastián Piñera, donde la integración social se posicionó como uno de los cinco ejes principales de planificación a nivel nacional, y se diseñaron los dos programas de vivienda con mezcla social vigentes: los Proyectos de Reactivación Económica e Integración Social (PREIS) y los Proyectos de Integración Social y Territorial (PIST).

En la primera etapa, se entendió la integración social como un desafío de inserción y localización (acceso a bienes y servicios, y conectividad), de 
mixtura social (cierto nivel de heterogeneidad social), de convivencia pacífica entre los habitantes, y de movilidad social ascendente (Ministerio de Vivienda y Urbanismo [MINVU], 2009). La cohesión social, en tanto, se asumió como una integración a nivel de ciudad y como condición para el desarrollo sostenible. Hacia comienzos del 2006, el MINVU se propuso trabajar en tres ejes ("Ciclo de reuniones", 2006): la cantidad, para terminar con el déficit del 20\% más pobre; la calidad, para revertir los bajos estándares de vivienda y barrio existentes; y la integración social, para disminuir los altos niveles de segregación,

Respecto de estos ejes entonces, la política del 2006 planteó una serie de mecanismos, los cuales Sabatini, Edwards, Cubillos, Brain, Mora, y Rasse (2010) clasifican en cuatro grupos. En el primero están los mecanismos dirigidos a mejorar la calidad, tanto de viviendas y barrios existentes, como de nuevos desarrollos. Los mecanismos hacia la vivienda existente incluyen; el Programa Quiero Mi Barrio, el Programa de Protección al Patrimonio Familiar, y una serie de modificaciones a los reglamentos del Fondo Solidario de Vivienda (FSV).
En el segundo grupo están los mecanismos para lograr una mejor localización, que incluyen el Subsidio a la Localización, el incentivo para compra de viviendas usadas, el Programa de Movilidad, y el Proyecto de Ley de Suelo e Integración Social. En el tercer grupo aparecen mecanismos para el aprovechamiento del suelo existente, apuntando a la flexibilización de los cambios de uso de suelo para instalar viviendas sociales, y a la gestión de suelos públicos para la vivienda social. Y en el cuarto grupo se identifican los programas que promueven la mezcla de grupos sociales distintos, aunque con limitadas exigencias de localización. Aquí hay mecanismos tales como los Proyectos de Desarrollo Urbano Condicionado (PDUC), y los Proyectos de Integración Social (PIS), desarrollados entre 2006 y 2013, y que ya no están vigentes. Institucionalmente, los PIS estaban sustentados en una mezcla informal de decretos, programas y reglamentos ${ }^{5}$. Estos decretos definen el máximo y mínimo de viviendas por subsidios, los montos de estos y los procedimientos de implementación, lo que está sintetizado en la Tabla 1.

5 De acuerdo con la Biblioteca Nacional del Congreso, los decretos son considerados como toda "Norma dictada por cualquier autoridad sobre los asuntos o negocios de su competencia. Cuando emana del presidente de la República se denomina Decreto Supremo". Recuperado de https://www.bcn.cl/ayuda_folder/glosario\#D. Los programas y reglamentos son documentos organizacionales, que establecen un conjunto de acciones, prestaciones y definiciones sobre una temática en particular. 
En la segunda etapa, la Política Nacional de Desarrollo Urbano, Ciudades Sustentables y Calidad de Vida, complementó la definición de integración propuesta por la política anterior, planteando que este concepto "debe relevarse en los mecanismos de gestión de suelo urbano y programas de vivienda" (Giménez y Ugarte, 2014, p. 31). Para alcanzar el ideal establecido, el MINVU planteó ocho macro-objetivos para trabajar, dentro de los que destacan "el establecer una política de suelo para promover la integración social" (Giménez y Ugarte, 2014, p. 30) como un mecanismo que se aleja de la lógica subsidiaria actual. Dentro de los mecanismos aparecen dos programas de mezcla social y que aún están vigentes. Primero están los Proyectos de Reactivación Económica con Integración Social (PREIS), cuyo reglamento se comenzó a diseñar bajo el primer gobierno de Sebastián Piñera y fue aprobado con el Decreto Supremo 116 del 2015 (DS116), durante el segundo gobierno de Bachelet. Y luego están los Proyectos de Integración Social y Territorial (PIST), aprobados por el Decreto Supremo 19 (DS19), también en el segundo gobierno de Bachelet el 2016 (Tabla 1).
Los PREIS son proyectos enmarcados dentro de la política de vivienda vigente y la misión institucional del MINVU, y buscan apoyar a las familias que no han podido materializar el subsidio habitacional adquirido, revertir la segregación socioespacial y contribuir a la reactivación económica (Fernández, Zamorano y Larenas, 2017). Los PIST por su parte, al ser considerados la continuidad de los PREIS, poseen los mismos objetivos y el mismo ítem presupuestario (Fernández et al., 2017). Sin embargo, estos poseen otros protocolos de implementación y montos de subsidios (Tabla 1). Tanto los PREIS como los PIST son considerados un avance en la claridad normativa de la mezcla social ("Primer informe", 2016), ya que los PIS no contaban con un decreto único.

Dos elementos incentivan la mezcla en estos proyectos. Primero, se entrega un Bono de Integración, que actúa como incentivo para los desarrolladores (aumentando su velocidad de venta), para las familias de clase media (entregando un beneficio económico), y para las familias pobres (entregando viviendas de mejor estándar) ("Primer informe", 2016). Segundo, se estipulan una serie de 


\section{TABLA 1. CARACTERÍSTICAS PRINCIPALES DE LOS PROGRAMAS ANALIZADOS}

\begin{tabular}{|c|c|c|c|}
\hline $\begin{array}{l}\text { DECRETO / } \\
\text { CARACTERÍSTICAS }\end{array}$ & $\begin{array}{l}\text { MEZCLA DE DECRETOS (DS40 + DS174 / } \\
\text { DS1 + DS49): PIS }\end{array}$ & DS116: PREIS & DS19: PIST \\
\hline $\begin{array}{l}\text { Porcentaje de vivienda } \\
\text { social }\end{array}$ & $\begin{array}{l}\text { DS40 + DS174: } 20 \% \text { mínimo y } 40 \% \text { máximo, } \\
\text { dependiendo de población comunal. } \\
\text { DS1 + DS49: } 30 \% \text { mínimo y } 70 \% \text { máximo. }\end{array}$ & $\begin{array}{l}10 \% \text { o } 20 \% \text { mínimo, dependiendo } \\
\text { del avance de la construcción, y } \\
40 \% \text { o } 60 \% \text { máximo, dependiendo } \\
\text { de población comunal. }\end{array}$ & $\begin{array}{l}20 \% \text { mínimo y } 40 \% \text { o } 60 \% \\
\text { máximo, dependiendo de } \\
\text { población comunal. }\end{array}$ \\
\hline $\begin{array}{l}\text { Valor máximo de } \\
\text { vivienda }\end{array}$ & $\begin{array}{l}\text { DS40 + DS174 } \\
\text { VS: } 750 \text { UF, } 950 \text { UF o } 1.000 \text { UF, dependiendo de } \\
\text { la región. } \\
\text { VSM: } 1.200 \text { UF o } 2.000 \text { UF, dependiendo de la } \\
\text { región. } \\
\text { DS1 + DS49 } \\
\text { VS: } 850 \text { UF, } 950 \text { UF o } 1.000 \text { UF, dependiendo de } \\
\text { la región y si es casa/departamento. } \\
\text { VSM: } 2.200 \text { UF o } 2.400 \text { UF, dependiendo de la } \\
\text { región y si es terreno propio. }\end{array}$ & $\begin{array}{l}\text { VS: } 900 \text { UF o } 1.000 \text { UF, dependien- } \\
\text { do de la región. } \\
\text { VSM: } 2.000 \text { UF o } 2.200 \text { UF, depen- } \\
\text { diendo de la región. }\end{array}$ & $\begin{array}{l}\text { VS: } 1.100 \text { UF o } 1.200 \text { UF, depen- } \\
\text { diendo de la región } \\
\text { VSM: } 2.200 \text { UF o } 2.400 \text { UF, } \\
\text { dependiendo de la región. }\end{array}$ \\
\hline $\begin{array}{l}\text { Año inicio decreto y } \\
\text { vigencia }\end{array}$ & $\begin{array}{l}\text { DS40 + DS174: } 2006 \text { (no vigente) } \\
\text { DS1 + DS49: } 2011 \text { (vigente). }\end{array}$ & 2014 (vigente). & 2016 (vigente). \\
\hline Bono de integración & $\begin{array}{l}\text { DS40 + DS174 } \\
\text { Aplicado a VSM: hasta } 100 \text { UF* }^{*} \\
\text { DS1 + DS49 } \\
\text { Aplicado a VSM: hasta } 100 \text { UF* }^{*}\end{array}$ & $\begin{array}{l}\text { VS: entre } 190 \text { UF y } 240 \text { UF, depen- } \\
\text { diendo de la región. } \\
\text { VSM: } 100 \text { UF. }\end{array}$ & $\begin{array}{l}\text { VSM: entre } 100 \text { UF y } 300 \text { UF, } \\
\text { dependiendo de familias } \\
\text { vulnerables en proyecto.* }\end{array}$ \\
\hline Foco principal & $\begin{array}{l}\text { DS40 + DS174: } \\
\text { Dar solución habitacional. } \\
\text { Favorecer integración social. } \\
\text { DS1 + DS49: } \\
\text { Favorecer integración y participación comuni- } \\
\text { taria. Reducir inequidad. }\end{array}$ & $\begin{array}{l}\text { Contribuir a reactivación } \\
\text { económica. } \\
\text { Favorecer integración social y } \\
\text { participación. } \\
\text { Reducir inequidad. }\end{array}$ & $\begin{array}{l}\text { Revertir déficit habitacional y } \\
\text { segregación. } \\
\text { Favorecer integración y } \\
\text { participación. } \\
\text { Reducir inequidad. }\end{array}$ \\
\hline $\begin{array}{l}\text { Proyecto emblemático } \\
\text { en Región Metropolita- } \\
\text { na, según MINVU }\end{array}$ & San Alberto de Casas Viejas (Puente Alto) & $\begin{array}{l}\text { Parque Cerro Negro (San } \\
\text { Bernardo). }\end{array}$ & Sin información \\
\hline
\end{tabular}


directrices de diseño que definen la homogeneidad física en todo el conjunto, reduciendo la diferencia entre grupos sociales (Maturana y Romano, 2015).

En la práctica de la planificación a nivel mundial, la premisa de la mezcla social es que la proximidad física entre diferentes grupos sociales puede facilitar la integración social (Ruiz-Tagle, 2013). Sin embargo, considerando los cinco conceptos claves descritos anteriormente (hipótesis del contacto, redes sociales, control social, modelos de rol y geografías de la oportunidad), los resultados de las ocho evaluaciones parciales de los PIS y PREIS que se han desarrollado hasta ahora y que son consideradas en este estudio, muestran una imagen bastante diferente. Respecto de la crítica a la hipótesis del contacto, la idea persistente de construir límites entre los habitantes de un mismo conjunto es una realidad dentro de los PIS. Maturana, Vergara y Romano (2016) señalan que hay conflictos, malestar, baja participación entre los vecinos e identificación de diferentes tipos de habitantes, los cuales están categorizados según su situación socioeconómica y de acuerdo a la modalidad de adquisición de la vivienda. Los vecinos se diferencian entre "los que pagan dividendos" y "los de atrás" (Maturana et al., 2016). En el caso de los PREIS, los conflictos entre residentes están marcados por el hecho de vivir en un condominio y compartir espacios comunes ("Primer informe", 2016). Los "lazos débiles" planteados por la idea de redes sociales no se logran formar en los PIS y los PREIS, ya que la convivencia y la distancia socioeconómica entre habitantes son bajas y no alcanzan para producir el capital social de puente para, por ejemplo, gatillar la contratación de servicios entre grupos. En el caso de los PIS, la mayoría de los habitantes de estos conjuntos, sin distinción de la modalidad de adquisición de vivienda, tiende a aislarse hacia el interior de su hogar, y la interacción es limitada (Maturana y Horne, 2016). Si bien la interacción es mayor en los PREIS, esta se ve limitada a temas de seguridad y a la organización de fiestas específicas ("Primer informe", 2016).

El control social está presente en los PIS y PREIS en el proceso de selección de nuevos residentes, lo que se transforma en exclusión. Las entidades desarrolladoras eligen a las familias vulnerables, considerando su estabilidad laboral y sus antecedentes penales ("Primer informe", 2016). Y en el caso de los PREIS, las reuniones informativas sobre el funcionamiento y reglas de la copropiedad, antes de la entrega de las viviendas, estuvieron exclusivamente orientadas a las familias pobres ("Primer informe", 2016). La idea de modelos de rol no se observa. En el caso de los PIS, no se identifica que exista un grupo social -sea este el de alto o bajo estatus- que dirija la comunidad a mejores comportamientos frente a las normas establecidas o a una mayor seguridad. Por ejemplo, la razón principal de los vecinos para mudarse es la percepción de comportamiento antisocial existente (Maturana et al., 2016). En relación con los PREIS, los vecinos no 
identifican el nivel socioeconómico como un factor de comportamiento socialmente aceptable y constructivo, sino la tenencia de un trabajo, independiente del ingreso que este genere ("Primer informe", 2016). Las geografías de oportunidad fueron observadas en al menos uno de los PIS. El conjunto habitacional Casas Viejas de Puente Alto consideró en su propuesta la incorporación de un colegio particular subvencionado (pagado y orientado a sectores medios), lo cual representó una ventaja comparativa de mercado, frente a otros proyectos habitacionales. Fuera de esto, resulta difícil que estos proyectos puedan atraer bienes y servicios de mayor calidad por la existencia de "grupos más poderosos" y transformarse así en una alternativa para mejorar las perspectivas de vida de los grupos vulnerables, ya que no existe una amplia diferencia socioeconómica entre los hogares beneficiarios.

En síntesis, los proyectos de mezcla social desarrollados en Chile no logran los objetivos de integración que plantea la retórica política, ya que dejan de lado procesos comunitarios y culturales, y niegan consideraciones de equidad, inclusión y convivialidad (Maturana y Horne, 2016). Y más aún, se ha observado que la proximidad entre los distintos grupos sociales ha generado en algunos casos nuevas formas de discriminación basadas en el clasismo (Ruiz-Tagle, 2016a). A pesar del escenario descrito por distintas investigaciones y de la falta de evidencia sobre la eficacia de estos proyectos, la asignación presupuestaria entre el 2016 y 2017 aumentó en un 155\% (Fernández et al., 2017) y se perfilan como uno de los mecanismos claves para la administración actual. Es decir, es esperable que la mezcla social en Chile pase de ser 'la excepción' a ser 'la regla'.

Desde un punto de vista más estructural, varios autores argumentan que los proyectos de vivienda de mezcla social son insuficientes frente a los problemas del mercado de suelo chileno (Dohnke, Heinrichs, Kabisch, Krellenberg, y Welz, 2015; Maturana et al., 2016). Tanto los PIS y PREIS como la vivienda social en general, están enfrentados a la competencia inmobiliaria de usos de suelo más rentables. Así, los montos de los subsidios no hacen frente a las alzas del suelo, con lo cual se reproduce el patrón periférico y no se puede promover desarrollos socialmente mixtos (Dohnke et al., 2015). Por último, se argumenta que no es moralmente justo subsidiar a la clase media, como ocurre con el Bono de Integración, cuando lo que supuestamente se quiere son mejores entornos residenciales para los más pobres (Sabatini, Mora, Polanco y Brain, 2014).

A pesar de todo lo anterior, la cuenta pública del primer gobierno de Bachelet por parte del Ministerio de Vivienda y Urbanismo (MINVU, 2010) plantea un diagnóstico muy condescendiente. El MINVU destaca el mejoramiento de la calidad de la gestión, de las viviendas y los barrios (nuevos y antiguos), una mayor cobertura frente al déficit 
(cuantitativo y cualitativo), y medidas frente a los desastres y frente a las crisis económicas. Sin embargo, la evaluación de las medidas de integración social está ausente y no hay ninguna mención al problema de la segregación, que era el foco principal del diagnóstico al iniciar el periodo. La localización sigue siendo un tema ambiguo, ya que de acuerdo con los parámetros de la pauta de aplicación y selección de proyectos, todo el Gran Santiago cumple con el requisito de localización. La cuenta pública del MINVU destaca el impacto positivo que estos proyectos generaron en el empleo y que se cambió el estándar de la vivienda social, lo cual es un logro bien merecido de esta administración. Pero esto solo refiere a la calidad, no a la localización ni tampoco a la integración.

\section{Conclusiones}

Es justo señalar que las políticas de vivienda en Chile desde el 2006 dieron un gran giro a lo que se venía haciendo: se reconocieron ampliamente los errores y se hizo un fuerte énfasis en mejorar la calidad. Pero a pesar de que los subsidios han sido reformulados e incrementados, se sigue descansando en los mismos mecanismos de mercado, en donde el acceso es facilitado por subsidios basados en la demanda (Dohnke et al., 2015). Así, tal como ocurre en todos los lugares del mundo en donde se han implementado políticas de mezcla social, se ha generado una gran discrepancia entre una retórica política que promete dar solución a décadas de segregación, y la realidad de unos instrumentos que no solo son insuficientes para lograr los ambiciosos objetivos sociales planteados, sino que además son inconsistentes con un contexto de libre mercado, con fuertes y crecientes desigualdades. Se reconoce que los proyectos de mezcla social han generado dos ganancias significativas: un aumento de la calidad de la vivienda y una percepción de mayor seguridad. Sin embargo, otros proyectos habitacionales, tales como "Parque Recoleta" (Recoleta) y "Altos de la Cordillera" (La Florida), han logrado los mismos beneficios sin utilizar la mezcla social como mecanismo.

La integración social se ha tomado la agenda periodística, política y académica chilena, sobre todo a partir de iniciativas recientes en comunas como Las Condes y Recoleta. Sin embargo, las políticas están tomando un rumbo muy similar al de otros países, y se visualizan al menos dos tipos de problemas. Por un lado, hay un 'pánico social' de los habitantes de clase media, ilustrado en el conflicto de Rotonda Atenas (Las Condes), y un 'pánico empresarial' del mundo inmobiliario, demostrado en las constantes reticencias de estos actores a participar de la mezcla social. Ante estos dos pánicos, y para continuar con las iniciativas, las políticas de muchos países han tendido a favorecer las 
expectativas morales de la clase media -a través de estrictos reglamentos de copropiedad, por ejemplo-, y a favorecer las expectativas de negocios del mundo privado, a través del aumento de subsidios, franquicias tributarias y/o facilidades administrativas. Así, las necesidades y expectativas de los pobres quedan en último lugar.

Por otro lado, hay un problema aún más preocupante: el aumento de los déficits habitacionales de los más pobres, que ha aparecido en todos los países en donde la mezcla social se ha implementado. En la medida que la mezcla social se convierte en 'la regla', los conjuntos que se construyen ya no son de 100\% vivienda social, sino solo de 30$40 \%$. Esto hace que se entreguen $60-70 \%$ menos de vivienda para los más pobres en cada conjunto construido. En Chile, los hogares allegados y los asentamientos informales han aumentado, el déficit habitacional ha subido (por distintas razones) en 8 de las actuales 15 regiones, hay muchos habitantes 'con subsidio y sin vivienda' (Rodríguez, Rodríguez, y Sugranyes, 2015) por la resistencia a ser relegados a la periferia lejana, y hay varias iniciativas cooperativistas planteando un camino propio para acceder a la vivienda, excluyendo al mercado de la ecuación tradicional de la política habitacional. Todo lo anterior hace pensar que el amplio consenso actual respecto de esta política, con actores que van desde el Partido Comunista hasta la derecha chilena, se podría ver socavado tanto por problemas de implementación como por resistencia ciudadana, como ha ocurrido a nivel internacional.

\section{Referencias bibliográficas}

Allport, G. (1954). The nature of prejudice. Cambridge, MA: Perseus Books.

Arthurson, K. (2002). Creating inclusive communities through balancing social mix: A critical relationship or tenuous link? Urban Policy and Research, 20(3), 245-261. https://doi. org/10.1080/0811114022000005898

Arthurson, K. (2010). Operationalising social mix: Spatial scale, lifestyle and stigma as mediating points in resident interaction. Urban Policy \& Research, 28(1), 49-63. https://doi. org/10.1080/08111140903552696

Arthurson, K. (2012a). Social mix and the city: Challenging the mixed communities consensus in housing and urban planning policies. Collingwood, Australia: CSIRO Publishing.

Arthurson, K. (2012b). Social Mix, reputation and stigma: Exploring residents' perspectives of neighbourhood effects. En M. van Ham, D. Manley, N. Bailey, L. Simpson, y D. Maclennan (Eds.), Neighbourhood effects research: New perspectives (pp. 101-120). New York, NY: Springer. https://doi. org/10.1007/978-94-007-2309-2_5 
Arthurson, K. (2013). Mixed tenure communities and the effects on neighbourhood reputation and stigma: Residents' experiences from within. Cities, 35, 432-438. https://doi.org/10.1016/j. cities.2013.03.007

Atkinson, R. y Kintrea, K. (2000). Owner-occupation, social mix and neighbourhood impacts. Policy \& Politics, 28(1), 93-108. https://doi. org/10.1332/0305573002500857

August, M. (2008). Social mix and Canadian public housing redevelopment: Experiences in Toronto. Canadian Journal of Urban Research, 17(1), 82-100.

August, M. (2014). Challenging the rhetoric of stigmatization: The benefits of concentrated poverty in Toronto's Regent Park. Environment and Planning A, 46(6), 1317-1333. https://doi.org/10.1068/a45635

Bacqué, M. h., Fijalkow, Y., Launay, L., y Vermeersch, S. (2011). Social mix policies in Paris: Discourses, policies and social effects. International Journal of Urban and Regional Research, 35(2), 256-273. https://doi.org/10.1111/j.1468-2427.2010.00995.x

Bolt, G. (2009). Combating residential segregation of ethnic minorities in European cities. Journal of Housing and the Built Environment, 24(4), 397-405. https://doi.org/10.1007/s10901-009-9164-y

Bolt, G., Özüekren, A. S., y Phillips, D. (2010). Linking integration and residential segregation. Journal of Ethnic and Migration Studies, 36(2), 169-186. https://doi.org/10.1080/13691830903387238.
Bolt, G., Phillips, D., y Van Kempen, R. (2010). Housing policy, (de)segregation and social mixing: An international perspective. Housing Studies, 25(2), 129-135. https://doi.org/10.1080/02673030903564838

Bolt, G. y van Kempen, R. (2013). Introduction special issue: Mixing neighbourhoods: success or failure? Cities, 35, 391-396. https://doi.org/10.1016/j. cities.2013.04.006

Borsdorf, A. e Hidalgo, R. (2008). New dimensions of social exclusion in Latin America: From gated communities to gated cities, the case of Santiago de Chile. Land Use Policy, 25(2), 153-160. https:// doi.org/10.1016/j.landusepol.2007.04.001

Bridge, G., Butler, T., y Le Galès, P. (2014). Power relations and social mix in metropolitan neighbourhoods in North America and Europe: Moving beyond gentrification? International Journal of Urban and Regional Research, 38(4), 1133-1141. https:// doi.org/10.1111/1468-2427.12125

Budds, J. y Teixeira, P. (2005). Ensuring the right to the city: pro-poor housing, urban development and tenure legalization in São Paulo, Brazil. Environment \& Urbanization, 17(1), 89-113. https://doi. org/10.1177/095624780501700105

Calavita, N. y Grimes, K. (1998). Inclusionary housing in California: The experience of two decades. Journal of the American Planning Association, 64(2), 150-169. https://doi.org/10.1080/01944369808975973 
Caldeira, T. (2000). City of walls: Crime, segregation, and citizenship in São Paulo. Berkeley, CA: University of California Press.

Cashin, S. (2004). The failures of integration: How race and class are undermining the American dream. New York, NY: Public Affairs.

Chaskin, R. y Joseph, M. (2010). Building "community" in mixed-income developments assumptions, approaches, and early experiences. Urban Affairs Review, 45(3), 299-335. https://doi. org/10.1177/1078087409341544

Chaskin, R., Khare, A., y Joseph, M. (2012). Participation, deliberation, and decision making the dynamics of inclusion and exclusion in mixed-income developments. Urban Affairs Review, 48(6), 863906. https://doi.org/10.1177/1078087412450151

Cheshire, P. (2007). Segregated neighbourhoods and mixed communities: A critical analysis. Joseph Rowntree Foundation.

Ciclo de reuniones: Políticas de suelo para la integración social. (2006). Recuperado de http:// politicaspublicas.uc.cl/publicacion/documentosconsejo-directivo-prourbana/i-ciclo-politicas-desuelo-para-la-integracion-social/

Cisneros, H. (2009). A new moment for people and cities. En H. Cisneros y L. Engdahl (Eds.), From despair to Hope: HOPE VI and the new promise of public housing in America's cities (pp. 3-14). Washington, DC: The Brookings Institution.

Deluca, S. y Rosenbaum, J. (2010). Residential mobility, neighborhoods and poverty: Results from the
Chicago Gautreaux program and the Moving to Opportunity experiment. En C. Hartman y G. Squires (Eds.), The integration debate: Competing futures for American cities (pp. 185-198). New York, NY: Routledge.

Dixon, J. (2001). Contact and boundaries: Locating the social psychology of intergroup relations. Theory \& Psychology, 11(5), 587-608. https://doi. org/10.1177/0959354301115001

Dohnke, J., Heinrichs, D., Kabisch, S., Krellenberg, K., y Welz, J. (2015). Achieving a socio-spatial mix? Prospects and limitations of social housing policy in Santiago de Chile. Housing Studies, 30(6), 839857. https://doi.org/10.1080/02673037.2014.9825 16

Ducci, M. E. (1997). Chile: El lado obscuro de una política de vivienda exitosa. EURE, 23(69), 99-115. Recuperado de http://www.eure.cl/index.php/eure/ article/view/1164

Ellen, I. G. (2000). Sharing America's neighborhoods. Cambridge, MA: Harvard University Press.

Fernández, V., Zamorano, H., y Larenas, J. (2017). Informe final de evaluación. Evaluación Programas gubernamentales (EPG). Programa Subsidio Habitacional Extraordinario Para Proyectos De Integración Social, DS No 116. Santiago, Chile: Gobierno de Chile, Direccion de Presupuestos. Recuperado de http:// www.dipres.gob.cl/597/articles-163124_informe_final.pdf

Fraser, J. y Kick, E. (2007). The role of public, private, non-profit and community sectors in shaping 
mixed-income housing outcomes in the US. Urban Studies, 44(12), 2357-2377. https://doi. org/10.1080/00420980701540952

Fraser, J., Oakley, D., y Levy, D. (2013). Guest editors introduction: Policy assumptions and lived realities of mixedincome housing on both sides of the Atlantic. Cityscape: A Journal of Policy Development and Research, 15(2), 1-14. Recuperado de https:// www.huduser.gov/portal/periodicals/cityscpe/ voll5num2/guest.html

Galster, G. (2013). Neighborhood social mix: Theory, Evidence, and implications for policy and planning. En N. Carmon y S. Fainstein (Eds.), Policy, planning and people: Promoting justice in urban development (pp. 307-336). Philadelphia, PA: University of Pennsylvania Press. https://doi. org/10.9783/9780812207965.307

Galster, G., Andersson, R., y Musterd, S. (2010). Who is affected by neighbourhood income mix? Gender, age, family, employment and income differences. Urban Studies, 47(14), 2915-2944. https://doi. org/10.1177/0042098009360233

Galster, G., Andersson, R., Musterd, S., y Kauppinen, T. (2008). Does neighborhood income mix affect earnings of adults? New evidence from Sweden. Journal of Urban Economics, 63(3), 858-870. https://doi.org/10.1016/j.jue.2007.07.002

Galster, G.y Killen, S. (1995). The geography of metropolitan opportunity: A reconnaissance and conceptual framework. Housing Policy Debate, 6(1), 7-43. https://doi.org/10.1080/10511482.1995.9521180
Giménez, P. y Ugarte, J.R. (Eds.). (2014). Hacia una nueva politica urbana para Chile. Politica nacional de desarrollo urbano: ciudades sustentables y calidad de vida. Santiago, Chile: PNUD, MINVU).

Goetz, E. (2013). The Audacity of HOPE VI: Discourse and the dismantling of public housing. Cities, 35, 342-348. https://doi.org/10.1016/j. cities.2012.07.008

Greenbaum, S. (2008). Report from the field: Social capital and deconcentration: Theoretical and policy paradoxes of the HOPE VI Program. North American Dialogue, 5(1), 9-13. https://doi.org/10.1525/ nad.2002.5.1.9

Greenbie, B. (1976). Design for diversity: Planning for natural man in the neo-technic environment: An ethological approach. New York, NY: Elsevier.

Hananel, R. (2014). Can centralization, decentralization and welfare go together? The case of Massachusetts Affordable Housing Policy (Ch. 40B). Urban Studies, 51(12), 2487-2502. https://doi. org/10.1177/0042098013512877

Hidalgo, R. (2004). La vivienda social en Santiago de Chile en la segunda mitad del siglo XX: Actores relevantes y tendencias espaciales. En C.d. Mattos, M.E. Ducci, A. Rodríguez, y G. Yañez (Eds.), Santiago en la globalización: ¿una nueva ciudad? (pp. 219-241). Santiago, Chile: Sur, Eure Libros.

Howell-Moroney, M. (2005). The geography of opportunity and unemployment: An integrated model of residential segregation and spatial mismatch. 
Journal of Urban Affairs, 27(4), 353-377. https://doi. org/10.1111/j.0735-2166.2005.00241.x

Imbroscio,D.(2008). "[U]nited and actuated bysomecommon impulse of passion": Challenging the dispersal consensus in American housing policy research. Journal of Urban Affairs, 30(2), 111-130. https:// doi.org/10.1111/j.1467-9906.2008.00381.x

Joseph, M. (2006). Is mixed-income development an antidote to urban poverty? Housing Policy Debate, 17(2), 209-234. https://doi.org/10.1080/10511482 .2006 .9521567

Joseph, M. (2008). Early resident experiences at a new mixed-income development in Chicago. Journal of Urban Affairs, 30(3), 229-257. https://doi. org/10.1111/j.1467-9906.2008.00394.x

Joseph, M. y Chaskin, R. (2010). Living in a mixed-income development: Resident perceptions of the benefits and disadvantages of two developments in Chicago. Urban Studies, 47(11), 2347-2366. https://doi.org/10.1177/0042098009357959

Kleinhans, R. (2004). Social implications of housing diversification in urban renewal: A review of recent literature. Journal of Housing and the Built Environment, 19(4), 367-390. https://doi.org/10.1007/ s10901-004-3041-5

Kleit, R. (2005). HOPE VI new communities: Neighborhood relationships in mixed-income housing. Environment and Planning A, 37(8), 1413-1441. https://doi.org/10.1068/a3796

Lemanski, C. (2006). Desegregation and integration as linked or distinct? Evidence from a previously 'White' suburb in post-apartheid Cape Town. International Journal of Urban and Regional Research, 30(3), 564-586. https://doi. org/10.1111/j.1468-2427.2006.00676.x

Lobo, B. (2015). Planning for urban renewal: Public/private initiatives in Bogota, Colombia, 2000-2014. Journal of Urban Regeneration \& Renewal, 8(3), 245-259.

Marcuse, P. (1997). The enclave, the citadel, and the ghetto. What has changed in the Post-Fordist US city. Urban Affairs Review, 33(2), 228-264. https:// doi.org/10.1177/107808749703300206

Maturana, B. y Horne, R. (2016). Towards socially integrated housing in Chile: Assessing conviviality through two key housing projects. Open House International, 41(2), 6-14.

Maturana, B. y Romano, S. (2015). Housing for social integration: Beyond physical proximity. Presentado en 31th International Conference PLEA 2015 Architecture in (R)Evolution Bologna, Italy.

Maturana, B., Vergara, L., y Romano, S. (2016). Vivienda pública de mixtura social en la ciudad neoliberal: Dinámicas de integración social en Villa Las Araucarias, La Serena, Chile. Presentado en Congreso Internacional Contested Cities, Madrid, España.

Ministerio de Vivienda y Urbanismo. (2009). Déficit urbano-habitacional: una mirada integral a la calidad de vida y el hábitat residencial en Chile. Santiago, Chile: Ministerio de Vivienda y Urbanismo.

Ministerio de Vivienda y Urbanismo. (2010). Cuenta pública 2006-2010. Santiago, Chile. Recuperado de 
http://www.minvu.cl/opensite_20091015151630. aspx

Muñoz, D. y Korthals, W. (2007). Lessons from Valencia: Separating infrastructure provision from land ownership. The Town Planning Review, 78(1), 6179. https://doi.org/10.3828/tpr.78.1.5

Musterd, S. y Andersson, R. (2005). Housing mix, social mix, and social opportunities. Urban Affairs Review, 40(6), 761-790. https://doi. org/10.1177/1078087405276006

Nyden, P., Maly, M., y Lukehart, J. (1997). The emergence of stable racially and ethnically diverse urban communities: A case study of nine US cities. Housing Policy Debate, 8(2), 491-534. https://doi.org/10 $.1080 / 10511482.1997 .9521262$

Primer informe. Estudio de casos de proyectos de integración social. Licitación ID 587-45-LE16. (2016). Santiago, Chile: Centro UC Políticas Públicas. Recuperado de https://politicaspublicas.uc.cl/wpcontent//uploads/2018/03/20161215-Informe-1_ minvu_final.pdf

Rodríguez, A., Rodríguez, P., y Sugranyes, A. (2015). Con subsidio, sin derecho. La situación del derecho a una vivienda adecuada en Chile. Santiago, Chile: SUR.

Rosenbaum, J., Stroh, L., y Flynn, C. (1998). Lake Parc Place: A study of mixed-income housing. Housing Policy Debate, 9(4), 703-740. https://doi.org/10.10 80/10511482.1998.9521314

Ruiz-Tagle, J. (2013). A theory of socio-spatial integration: Problems, policies and concepts from a US perspective. International Journal of Urban and
Regional Research, 37(2), 388-408. https://doi. org/10.1111/j.1468-2427.2012.01180.x

Ruiz-Tagle, J. (2016a). La persistencia de la segregación y la desigualdad en barrios socialmente diversos: un caso de estudio en la Florida, Santiago. EURE, 42(125), 81-108. https://doi.org/10.4067/ s0250-71612016000100004

Ruiz-Tagle, J. (2016b). The broken promises of social mix: The case of the Cabrini Green - Near North area in Chicago. Urban Geography, 37(3), 352-372. https://doi.org/10.1080/02723638.2015.1060697

Ruming, K., Mee, K., y McGuirk, P. (2004). Questioning the rhetoric of social mix: Courteous community of hidden hostility. Australian Geographical Studies, 42(2), 234-248. https://doi. org/10.1111/j.1467-8470.2004.00275.x

Sabatini, F., Cáceres, G., y Cerda, J. (2001). Segregación residencial en las principales ciudades chilenas: Tendencias de las tres últimas décadas y posibles cursos de acción. EURE, 27(82), 21-42. https://doi. org/10.4067/s0250-71612001008200002

Sabatini, F., Edwards, G., Cubillos, G., Brain, I., Mora, P., y Rasse, A. (2010). Dispersión espacial de vivienda económica como vía de integración social urbana. En Camino al Bicentenario: propuestas para Chile. Concurso politicas públicas (pp. 247-273). Santiago, Chile: Pontificia Universidad Católica de Chile.

Sabatini, F., Mora, P., Polanco, I., y Brain, I. (2014). Conciliando integración social y negocio inmobiliario: Seguimiento de proyectos de integración 
social (PIS) desarrollados por inmobiliarias e implicancias de política. En M. Smolka y F. Furtado (Eds.), Instrumentos notables de políticas de suelo en América Latina (pp. 61-67). Quito, Ecuador: Lincoln Institute of Land Policy.

Salcedo, R. y Torres, A. (2004). Gated communities in Santiago: Wall or frontier? International Journal of Urban and Regional Research, 28(1), 27-44. https:// doi.org/10.1111/j.0309-1317.2004.00501.x

Sarkissian, W. (1976). The idea of social mix in town planning: An historical review. Urban Studies, 13(3), 231-246. https://doi. org/10.1080/00420987620080521

Sarkissian, W., Forsyth, A., y Heine, W. (1990). Residential "social mix": The debate continues. Australian Planner, 28(1), 5-16. https://doi.org/10.1080/0729 3682.1990.9657439

Siclari, P. (2009). Instrumentos de integración social en la nueva Política Habitacional y Urbana de Chile. Centro-h, Revista de la Organización Latinoamericana y del Caribe de Centros Históricos, 3(1), 63-75. Recuperado de https://www.redalyc.org/ pdf/1151/115112536006.pdf

Silver, H. (2013). Mixing policies: Expectations and achievements. Cityscape: A Journal of Policy Development and Research, 15(2), 73-82. Recuperado de https://www.huduser.gov/portal/periodicals/ cityscpe/vol15num2/article5.html

Smeets, P. y Salman, T. (2008). Countering urban segregation: Theoretical and policy innovations from around the globe. Urban Studies, 45(7), 1307-1332. https://doi.org/10.1177/0042098008090676

Smith, J. (2006). Mixed-income communities: Designing out poverty or pushing out the poor? En L. Bennett, J. Smith, y P. Wright (Eds.), Where are poor people to live (pp. 259-281). Armonk, NY: M.E. Sharpe.

Smith, J. (2010). Integration: Solving the wrong problem. En C. Hartman y G. Squires (Eds.), The integration debate: Competing futures for American cities (pp. 229-245). New York, NY: Routledge.

Smith, R. (1998). Discovering stable racial integration. Journal of Urban Affairs, 20(1), 1-25. https://doi. org/10.1111/j.1467-9906.1998.tb00407.x

Sugranyes, A. (2005). La política habitacional en Chile, 1980-2000: Un éxito liberal para dar techo a los pobres. En A. Rodríguez y A. Sugranyes (Eds.), Los con techo: Un desafio para la politica de vivienda social (pp. 23-57). Santiago, Chile: Ediciones SUR.

Svampa, M. (2001). Los que ganaron. La vida en los countries y en los barrios privados. Buenos Aires, Argentina: Editorial Biblos.

Tach, L. (2009). More than bricks and mortar: Neighborhood frames, social processes, and the mixedincome redevelopment of a public housing project. City \& Community, 8(3), 269-299. https://doi. org/10.1111/j.1540-6040.2009.01289.x

Talen, E. (2008). Design for diversity: Exploring socially mixed neighborhoods. Burlington, MA: Elsevier. 
Uitermark, J. (2014). Integration and control: The governing of urban marginality in Western Europe. International Journal of Urban and Regional Research, 38(4), 1418-1436. https://doi. org/10.1111/1468-2427.12069

Van Ham, M. y Manley, D. (2010). The effect of neighbourhood housing tenure mix on labour market outcomes: A longitudinal investigation of neighbourhood effects. Journal of Economic Geography, 10(2), 257-282. https://doi.org/10.1093/jeg/ lbp017

Vischer, J. (1986a). Social mix and environmental design: Exploration of a contemporary concept. Journal of Architectural and Planning Research, 3(4), 315-326. Recuperado de https://www.jstor.org/ stable/43028820

Vischer, J. (1986b). The complexity of designing for social mix: An evaluation of site-planning principles. Journal of Architectural and Planning Research, 3(1), 15-32. Recuperado de https://www.jstor.org/ stable/43028785

Wacquant, L. (1997). Three pernicious premises in the study of the American ghetto. International Journal of Urban and Regional Research, 21(2), 341-353. https://doi.org/10.1111/1468-2427.00076

Wirth, L. (1927). The ghetto. American Journal of Sociology, 33(1), 57-71. https://doi.org/10.1086/214333 